\title{
Spontaneous Partial Regression of Cerebral Arteriovenous Malformation
}

\author{
Jae Ho Choi, MD' \\ Ji Hoon Shin, MD' \\ Seong Shik Cho, MD' \\ Deuk Lin Choi, MD' \\ Bark Jang Byun, $\mathrm{MD}^{2}$ \\ Dong Won Kim, MD
}

\begin{abstract}
Arteriovenous malformation (AVM) of the brain is one of the important pathologic conditions which cause intracerebral or subarachnoid hemorrhage, epilepsy, or chronic cerebral ischemia. The spontaneous regression of cerebral AVM is reported to be very rare and more likely to occur when the AVM is small, is accompanied by hemorrhage, and has fewer arterial feeders. We report a case of right occipital AVM which at follow-up angiography performed four years later showed near-complete spontaneous regression.
\end{abstract}

Index terms:

Brain, abnormalities

Brain, diseases

Cerebral blood vessels, abnormalities

Korean J Radiol 2002; 3:74-77

Received September 13, 2001; accepted after revision January 21, 2002.

Departments of ${ }^{1}$ Radiology, ${ }^{2}$ Neurosurgery, and ${ }^{3}$ Pathology, Soonchunhyang University Hospital, Soonchunhyang University College of Medicine

Address reprint requests to: Ji Hoon Shin, MD, Department of Radiology, Soonchunhyang University Hospital, 657 Hannam-dong, Yongsan-gu, Seoul 140-743, South Korea. Telephone : (822) 709-9396 Fax : (822) 795-3928 e-mail : jhshin@hosp.sch.ac.kr
7 he complete or partial spontaneous regression of a cerebral AVM is a very rare event, with a reported frequency of 0.8 to $20 \%$ of the total number of reported cases (1-3). In most of these, a hemorrhagic event or surgical manipulation will have contributed to the thrombosis of the AVM (1-4). We describe a case in which spontaneous regression of a right occipital AVM, initially manifesting as an intracerebral hemorrhage, occurred.

\section{CASE REPORT}

At initial presentation, a 41-year-old man complained of headache and blurred vision during exercise. The results of neurological examination were unremarkable, but contrast-enhanced CT scanning demonstrated a large intracerebral hematoma in the right occipital lobe accompanied by enlarged and tortuous vascular structures (Fig. 1A). At contrast enhanced T1-weighted MR imaging, in addition to the hematoma, a definite vascular structure representing an AVM was seen (Fig. 1B). Vertebral angiography revealed that this was supplied by the right posterior cerebral artery and drained into the right transverse sinus (Figs. 1C, D). Angiography of the internal carotid artery revealed no arterial feeder, and the external carotid was not angiographically examined at that time. The patient declined surgical treatment, including intravascular embolization. Four years later, although almost asymotomatic, he was admitted for treatment of the AVM. Follow-up contrast enhanced CT and cerebral angiography showed near-complete disappearance of the previous AVM, with normalization of its feeder, the right posterior cerebral artery (Figs. 2A, B). A residual AVM was revealed by right external carotid angiography (Figs. 2C, D); during the delayed phase of this, the draining vein seen during previous vertebral angiography was opacified. The lesion was removed surgically and confirmed as an AVM with multiple thrombotic foci (Fig. 2E). 


\section{DISCUSSION}

Cerebral AVM occurs in approximately $0.14 \%$ of the general population, the most common presenting symptoms being hemorrhage and seizure. AVM is a complex network of vascular channels consisting of arterial feeders, an AVM nidus, and enlarged venous outflow channels, all of which are demonstrable at angiography. In our case, the AVM was located in the right occipital region. It was supplied by a single arterial feeder (the right posterior cerebral artery) and drained through a single vein into the right transverse sinus. The natural history of AVMs has been extensively described, and usually includes enlargement and hemorrhage but rarely regression (5). It has been speculat- ed that the spontaneous disappearance of a cerebral AVM could be caused by thrombosis associated with hemorrhage or surgical manipulation; decreased flow associated with the elongation of abnormal vessels secondary to compression by an intracerebral clot or edema, turbulence, or emboli; or intrinsic changes in the vessel wall leading to hypercoagulability $(1,2,4,6)$. When regression does occur, it is therefore necessary to identify the associated factors, which may include subarachnoid hemorrhage, subdural or intracerebral hematoma, brain tumor, atherosclerosis, surgery, radiation therapy, or oral contraceptive use $(1,2$, $4,6-8)$. In a case involving of associated hemorrhage in or around a cerebral AVM, the hemorrhage will induce local hemodynamic alteration, decreased blood flow, and subsequent thrombosis by exerting a mass effect due to the clot-

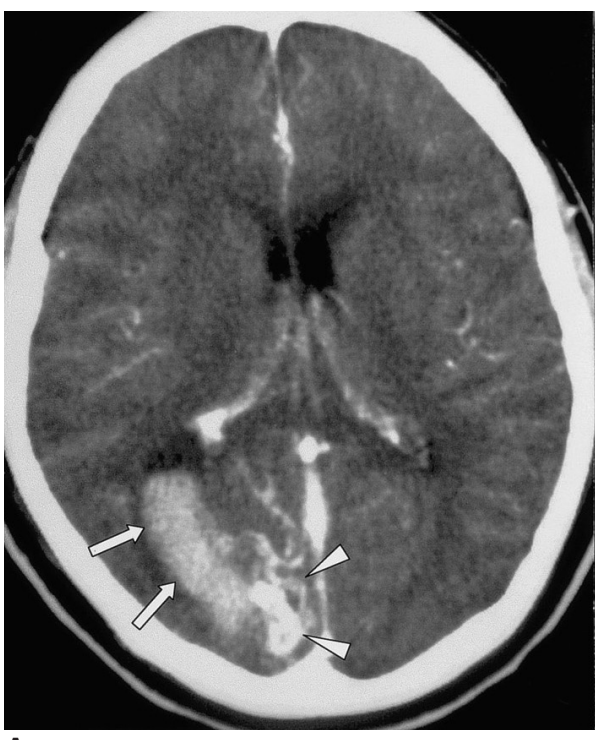

A

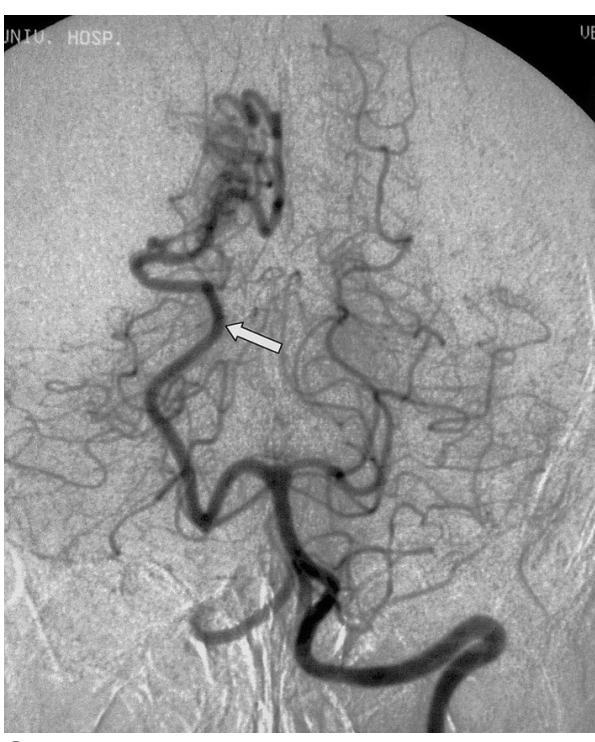

C

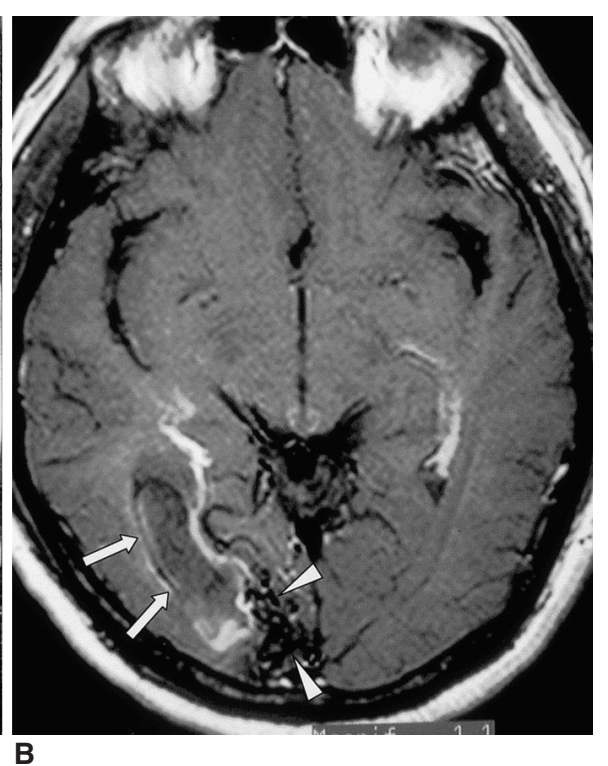

B

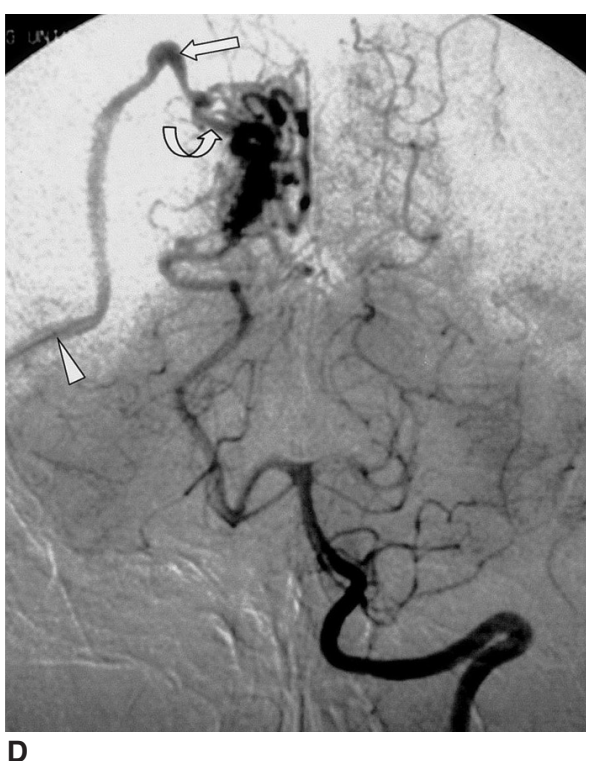

Fig. 1. Initial radiologic studies of an AVM in a 41-year-old man with sudden onset of headache and blurred vision.

A. Enhanced CT scan shows a large intracerebral hematoma (arrows) in the right occipital lobe. Clearly enhanced tubular structures (arrowheads) are seen in the medial occipital lobe adjacent to the hematoma.

B. Axial gadolinium-enhanced T1weighted MR image suggests that the hematoma i.e. is not certain; thus, 'suggests that is iso-intense, with a peripheral high-signal-intensity rim (arrows) secondary to the presence of methemoglobin'. The tubular structures representing the AVM are seen as signal voids (arrowheads).

C. Early-phase left vertebral angiogram depicts an AVM supplied by the right posterior cerebral artery (arrow).

D. Delayed-phase left vertebral angiogram shows an early-draining single vein (arrow) which drains into the right transverse sinus (arrowhead). Note the tortuous proximal portion of the early draining vein (curved arrow). 
ting of blood and surrounding edema $(1,5)$. In our case, pathologic examination revealed prominent thrombosis within the vessels; Minakawa et al. (2), on the other hand, reported that smaller, superficial AVMs with a single or a few feeders in older patients (more than 30 years of age) had a greater propensity to thrombose and regress while in younger patients, larger, deep AVMs with multiple arterial feeders tended to increase in size during the follow-up period. The circumstances of our present case are consistent with the observations of Minakawa et al. in that the AVM is relatively small and had only a single arterial feeder, and the patient is more than 30 years old. In addition, our patient had a single draining vein which might be comparable to a single arterial feeder in that an AVM with a single draining vein would be more affected by hemodynamic changes secondary to hematoma or edema than one with multiple draining veins. This is supported by a review of the literature carried out by Krapf et al.(9), who pointed out that a single draining vein was found in $84 \%$ of cases in which there was spontaneous occlusion of a cerebral AVM, while a single feeder was found in only $30 \%$, implying that the draining vein may be more important. Interestingly, in our case, the proximal portion of the draining vein seen at initial vertebral angiography was no longer seen at follow-up external carotid angiography, although the distal portion of this vein was the same in both instances. This finding strongly suggests that thrombosis occurred in the proximal part of the draining vein during fol-
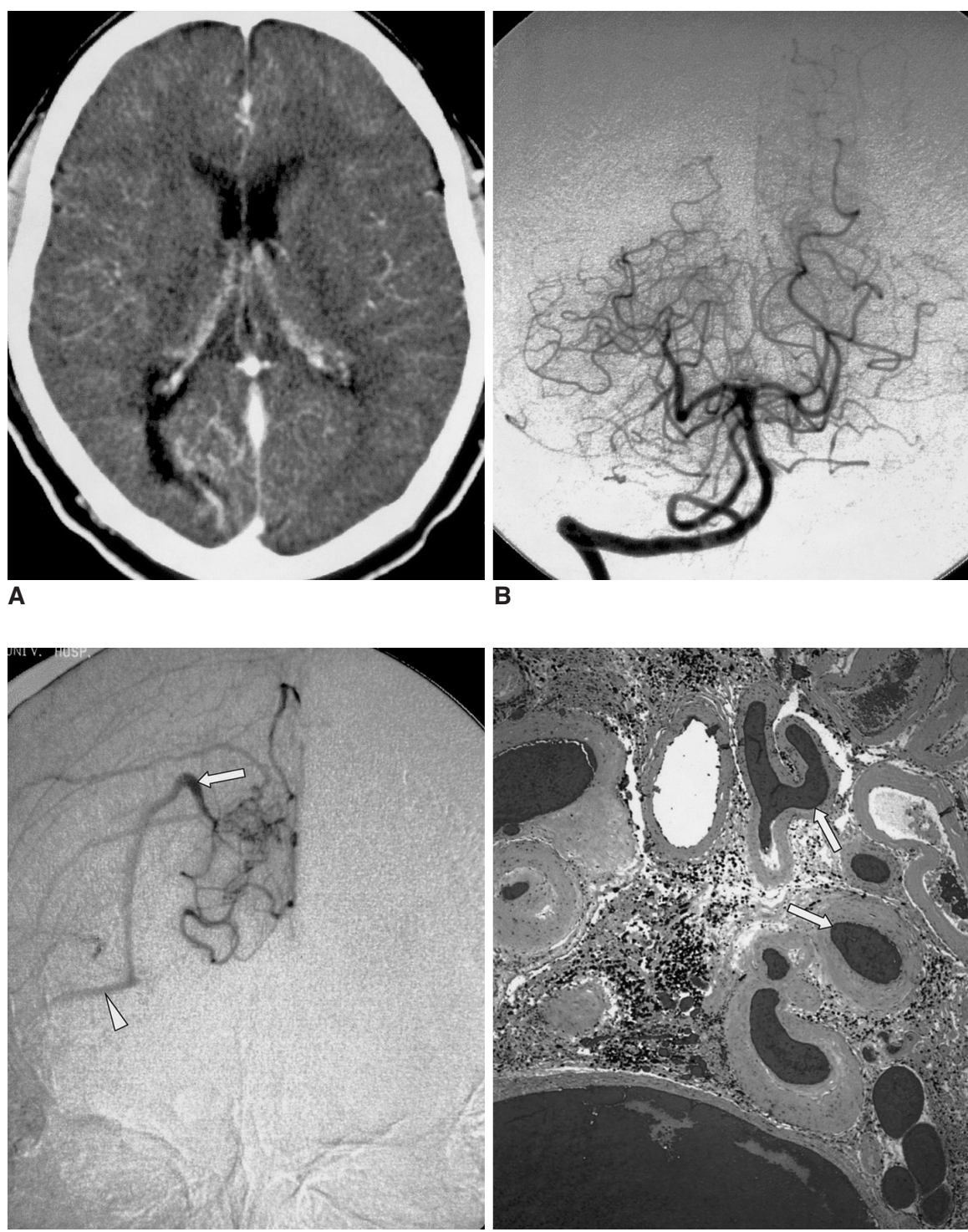

D

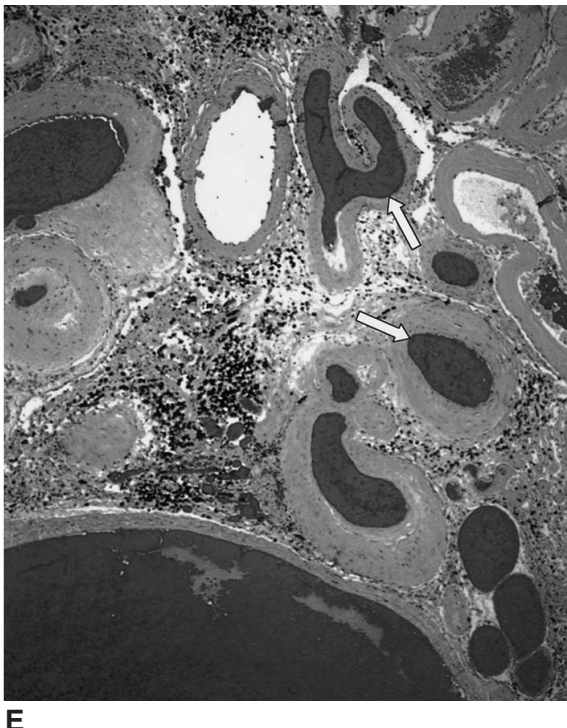

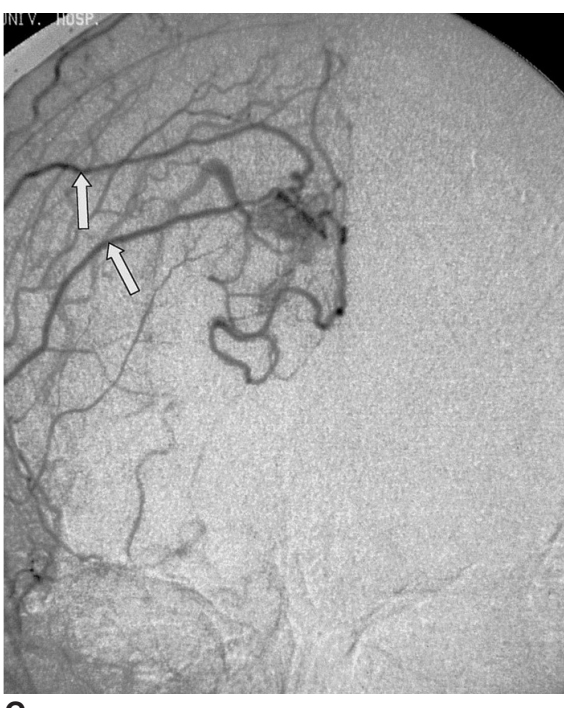

C

Fig. 2. Follow-up CT scan and angiogram four years later without further treatment.

A. Enhanced CT scan reveals marked regression, with a small residual AVM in the right occipital lobe and some encephalomalatic change at the site of the previous hematoma.

B. Right vertebral angiography shows complete disappearance of the AVM in the right occipital lobe.

C. Early-phase angiogram of the right external carotid artery depicts a residual AVM supplied by the right middle meningeal artery (arrows).

D. Late-phase angiogram of the right external carotid artery shows the same draining vein (arrow) seen at initial vertebral angiography, which drains into the right transverse sinus (arrowhead). The disappearance of the previous proximal portion of the draining vein seen in Fig. 1D suggests thrombus formation.

E. Photomicrograph (hematoxylin-eosin stain; magnification $\times 100$ ) depicts an AVM composed of abnormal vessels of various sizes and with fresh intraluminal thrombi (arrows). 


\section{Spontaneous Partial Regression of Cerebral Arteriovenous Malformation}

low-up, although whether or not spontaneous regression of the pial supply is accompanied by the development of the dural component cannot be determined. Unfortunately, the changes seen at external carotid angiography could not be assessed because initial angiography had not included this procedure.

In conclusion, in patients scheduled for surgical treatment of an AVM diagnosed several months or years earlier, imaging studies such as angiography or CT must be performed in order to assess whether spontaneous regression of the AVM, which is rare, has occurred.

\section{Acknowledgments}

We are very grateful to Bonnie Hami, M.A., Department of Radiology, University Hospitals of Cleveland, U.S.A., for her editorial assistance.

\section{References}

1. Patel MC, Hodgson TJ, Kemeny AA, Forster DM. Spontaneous obliteration of pial arteriovenous malformations: a review of 27 cases. Am J Neuroradiol 2001;22:531-536

2. Minakawa T, Tanaka R, Koike T, Takeuchi S, Sasaki O. Angio- graphic follow-up study of cerebral arteriovenous malformations with reference to their enlargement and regression. Neurosurgery 1989;24:68-74

3. Abdulrauf SI, Malik GM, Awad IA. Spontaneous angiographic obliteration of cerebral arteriovenous malformations. Neurosurgery 1999;44:280-288

4. Gibb PA, Gullan RW, Sandhu N. Spontaneous angiographic disappearance of an intracranial arteriovenous malformation. $\mathrm{Br} J$ Neurosurg 1988;2:529-533

5. Chen JW, Kerber C, U HS. Spontaneous regression of large bilateral basal ganglia arteriovenous malformations. AJNR 1991; 12:835-837

6. Omojola MF, Fox AJ, Vinuela FV, Drake CG. Spontaneous regression of intracranial arteriovenous malformations: Report of three cases. J Neurosurg 1982;57:818-822

7. Hamada J, Yonekawa Y. Spontaneous disappearance of a cerebral arteriovenous malformation: case report. Neurosurgery 1994;34:171-173

8. Sartor K. Spontaneous closure of cerebral arteriovenous malformation demonstrated by angiography and computed tomography. Neuroradiology 1978;15:95-98

9. Krapf H, Siekmann R, Freudenstein D, Kuker W, Skalej M. Spontaneous occlusion of a cerebral arteriovenous malformation: Angiographic and MR imaging follow-up and review of the literature. AJNR 2001;22:1556-1560 